\title{
Reduced tolerance to abiotic stress in transgenic Arabidopsis overexpressing a Capsicum annuum multiprotein bridging factor 1
}

\author{
Wei-Li Guo ${ }^{1,3+}{ }^{\text {, Ru-Gang Chen }}{ }^{1,2 \dagger}$, Xiao-Hua Du ${ }^{3}$, Zhen Zhang ${ }^{1}$, Yan-Xu Yin ${ }^{1}$, Zhen-Hui Gong ${ }^{1,2^{*}}$ \\ and Guang-Yin Wang ${ }^{3}$
}

\begin{abstract}
Background: The pepper fruit is the second most consumed vegetable worldwide. However, low temperature affects the vegetative development and reproduction of the pepper, resulting in economic losses. To identify cold-related genes regulated by abscisic acid (ABA) in pepper seedlings, cDNA representational difference analysis was previously performed using a suppression subtractive hybridization method. One of the genes cloned from the subtraction was homologous to Solanum tuberosum MBF1 (StMBF1) encoding the coactivator multiprotein bridging factor 1. Here, we have characterized this StMBF1 homolog (named CaMBF1) from Capsicum annuum and investigated its role in abiotic stress tolerance.

Results: Tissue expression profile analysis using quantitative RT-PCR showed that CaMBF1 was expressed in all tested tissues, and high-level expression was detected in the flowers and seeds. The expression of CaMBF1 in pepper seedlings was dramatically suppressed by exogenously supplied salicylic acid, high salt, osmotic and heavy metal stresses. Constitutive overexpression of CaMBF1 in Arabidopsis aggravated the visible symptoms of leaf damage and the electrolyte leakage of cell damage caused by cold stress in seedlings. Furthermore, the expression of RD29A, ERD15, KIN1, and RD22 in the transgenic plants was lower than that in the wild-type plants. On the other hand, seed germination, cotyledon greening and lateral root formation were more severely influenced by salt stress in transgenic lines compared with wild-type plants, indicating that CaMBF1-overexpressing Arabidopsis plants were hypersensitive to salt stress.
\end{abstract}

Conclusions: Overexpression of CaMBF1 in Arabidopsis displayed reduced tolerance to cold and high salt stress during seed germination and post-germination stages. CaMBF1 transgenic Arabidopsis may reduce stress tolerance by downregulating stress-responsive genes to aggravate the leaf damage caused by cold stress. CaMBF1 may be useful for genetic engineering of novel pepper cultivars in the future.

Keywords: Capsicum annuum L, Cold stress, Salt stress, CaMBF1, Arabidopsis

\section{Background}

Transcriptional regulatory proteins play a central role in the expression of genomic information during complex biological processes in all organisms. Among these proteins, transcriptional co-activators are key components of eukaryotic gene expression by interacting with both

\footnotetext{
*Correspondence: zhgong@nwsuaf.edu.cn

${ }^{\dagger}$ Equal contributors

${ }^{1}$ College of Horticulture, Northwest A\&F University, Yangling, Shaanxi, P R China

${ }^{2}$ State Key Laboratory of Crop Stress Biology in Arid Areas, Northwest A\&F

University, Yangling, Shaanxi, P R China

Full list of author information is available at the end of the article
}

transcription factors and/or other regulatory elements and the basal transcription machinery [1,2]. Multiprotein bridging factor 1 (MBF1), a transcriptional co-activator, enhances transcription of its target genes by bridging the general factor TBP (TATA box Binding Protein) and specific transcription factors bound to their target promoters in eukaryotes such as yeast [3], Drosophila [4] and Arabidopsis [5].

MBF1-type genes (SlER24 and StMBF1) encode functional transcriptional co-activators as demonstrated by their capacity to complement the yeast mbf1 mutant 
[6,7]. Fusion of tomato SIER24 to EAR (Amphiphilic Repression) in the MicroTom cultivar induced a delay of seed germination, but had no obvious effect on plant growth [6]. Moreover, it was reported that the StMBF1 gene in potato was induced by pathogen attack, oxidative stress, wounding and in response to salicylic acid (SA) treatment $[7,8]$. Direct evidence of the involvement of $M B F 1$ in plant responses to environmental stresses was obtained by enhancing tolerance to heat and osmotic stresses in transgenic Arabidopsis lines expressing the $A t M B F 1 c$ gene and more recently AtMBF1a, without growth retardation $[9,10]$. These data indicate that $M B F 1$-like genes can be associated with a variety of developmental processes in plants such as environmental stress tolerance. To date, there are very few data on the significance of $M B F 1$ in cold stress tolerance.

Pepper (Capsicum annuum L.) is a member of the Solanaceae family, and an important vegetable and spice crop valued for its aroma, taste, pungency and flavor. The pepper fruit is the second most consumed vegetable around the world [11]. Different types of peppers, including chili, mild and sweet peppers are cultivated worldwide. Low temperature is one of the most important abiotic factors limiting the growth, development and geographical distribution of plants [12]. Pepper plants originate from tropical regions and are very sensitive to low temperature, which affects their vegetative development and reproduction, resulting in economic losses [13-15]. As part of production and fruit quality improvement, we are interested in investigating plant defense mechanisms to improve resistance to environmental stresses. In our previous report, we showed that exogenous application of ABA increased the tolerance of pepper seedlings to chilling-induced oxidative damage, mainly by enhancing the activity of antioxidant enzymes and expression of related genes [16]. Furthermore, ABAmediated candidate genes associated with chilling stress have been fully characterized in pepper plants using a suppression subtractive hybridization (SSH) method [17]. One of the genes cloned from the reverse subtraction was homologous to Solanum tuberosum MBF1 (StMBF1) encoding the coactivator multiprotein bridging factor 1 . Expression of this $M B F 1$ homologue was highly induced by cold stress, whereas ABA-pretreatment decreased its expression in pepper seedlings subjected to cold stress. However, the function of this gene involved in the defense response to chilling stress remains to be elucidated.

In this study, based on the above-mentioned expressed sequence tag (EST) from the reverse SSH library that enriched the up-regulated expressed genes responding to chilling stress, we have functionally characterized the homolog of StMBF1 in pepper (designated as CaMBF1). The results of this study suggest that CaMBF1 transcript in pepper seedlings can be suppressed by SA, salt, osmotic and heavy metal stresses. Overexpression of
CaMBF1 in Arabidopsis displayed reduced tolerance to cold and high salt stress.

\section{Results}

Isolation of the CaMBF1 CDNA clone and sequence analysis A differential screening of a cold-related pepper seedling cDNA library, using PCR-amplified subtracted and control probes, was performed previously [17]. One of the isolated clones exhibited $80 \%$ identity at the nucleotide level to StMBF1 from Solanum tuberosum [8]. A full-length clone of this homologue was obtained by a homology-based candidate gene method, including the complete open reading frame. The gene was named CaMBF1 and submitted to GenBank with the Accession Number JX402927. The size of the CaMBF1 clone was $648 \mathrm{bp}$, comprising an open reading frame of $420 \mathrm{bp}$ (139 amino acids). The predicted polypeptide was basic, with a pI of 9.86 and a molecular mass of $15.3 \mathrm{kDa}$. An alignment of the deduced amino acid sequence of $C a M B F 1$ with other homologous sequences is presented in Figure 1. At the amino acid level, CaMBF1 showed a high degree of conservation with known genes of other plant species: Solanum tuberosum (StMBF1, 95\% identity) [8], and Arabidopsis thaliana (AtMBF1b, 80\% identity; AtMBF1a, 79\% identity) [10].

\section{Expression of CaMBF1 in pepper seedlings is severely suppressed by stress and SA treatments}

A number of $M B F 1$ genes were found to be differentially induced by abiotic stress $[10,18,19]$. Therefore, we suspected that the $C a M B F 1$ gene may be involved in stress signaling pathways and were interested in its possible function in stress responses. As a first step toward functional analysis, we examined the expression pattern of CaMBF1 in pepper plants using qRT-PCR analysis. This analysis revealed that the $C a M B F 1$ gene was expressed ubiquitously in all developmental stages of plants and in all tested organs, including root, stem, leaf, flower, fruit and seed (Figure 2). High-level expression was detected in flower and seed, although expression level in root was rather low. As shown in Figure 3, CaMBF1 expression was dramatically decreased by several stress conditions, including $5 \mathrm{mM}$ $\mathrm{SA}$, high salt (300 mM NaCl), osmotic stress (300 mM mannitol), and heavy metal (300 $\mu \mathrm{M} \mathrm{Hg})$. Rapid and robust down-regulation of CaMBF1 transcript was observed at $1 \mathrm{~h}$ after salt, osmotic and heavy metal treatments, which decreased to 0.06-fold, 0.03-fold and 0.12-fold, respectively. In contrast, a slight reduction of CaMBF1 transcript was found during $12 \mathrm{~h}$ of SA treatment and followed by an increase to the initial level (Figure 3A).

\section{Reduced tolerance of CaMBF1-overexpressing Arabidopsis plants to cold stress}

To test the function of CaMBF1 in Arabidopsis, we generated transgenic plants that constitutively expressed 


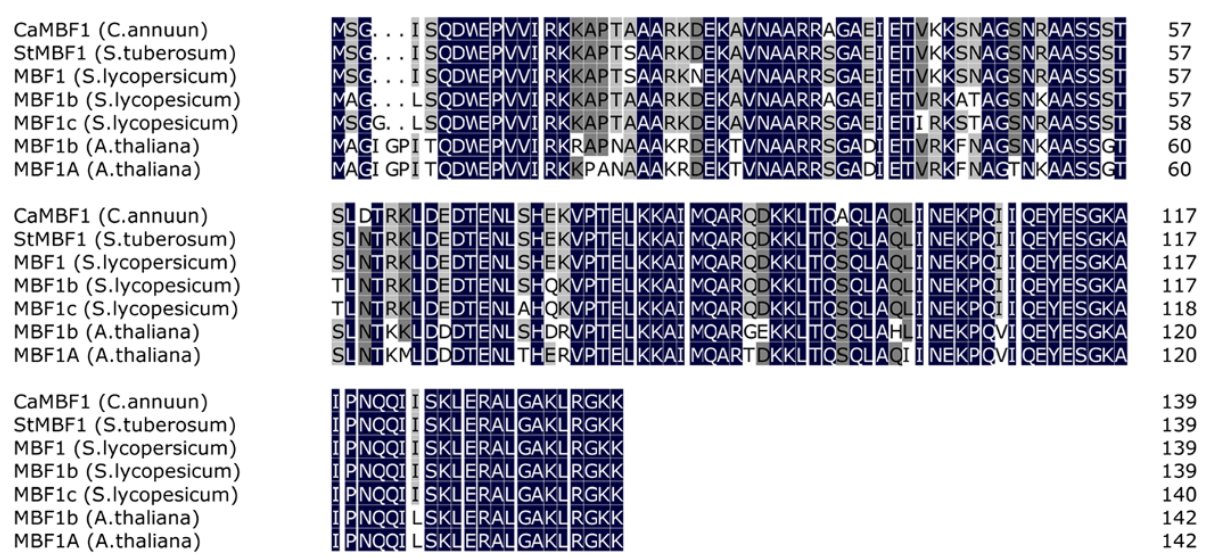

Figure 1 Alignment of deduced amino acid sequences of CaMBF1 and other MBF proteins. StMBF1 (AAF81108.1) from Solanum tuberosum, MBF1 (NP_001234341.1), MBF1b (XP_004251896.1), MBF1c (ABG29114.1) from Solanum lycopersicum and MBF1A (NP_565981.1), MBF1b (NP_191427.1) from Arabidopsis thaliana. Conserved residues are shaded in black, dark grey shading indicates similar residues in at least six out of the seven sequences, and light grey shading indicates similar residues in four to five out of the seven sequences.

CaMBF1 under the control of the CaMV $35 S$ promoter. Transgenic plants expressing CaMBF1 appeared similar in their growth and development to WT plants. However, as shown in Figure 4, the transgenic plants were larger than the WT plants during the florescence production period; the rosette leaves of transgenic plants were $70 \%$ longer and 60\% wider than those of WT plants.

To study the response of CaMBF1-expressing plants to abiotic stress, 2-week-old WT and transgenic seedlings were subjected to several stresses, including cold, salinity, and ABA. Firstly, transcript levels of the high homology (AtMBF1a, AtMBF1b or AtMBF1c) modulated by the overexpression of $C A M B F 1$ under normal conditions were determined by qRT-PCR. Compared to WT plants, the expression of the homologous genes was not basically altered in transgenic plants when grown in normal condition (Figure 5), indicating that overexpression of pepper

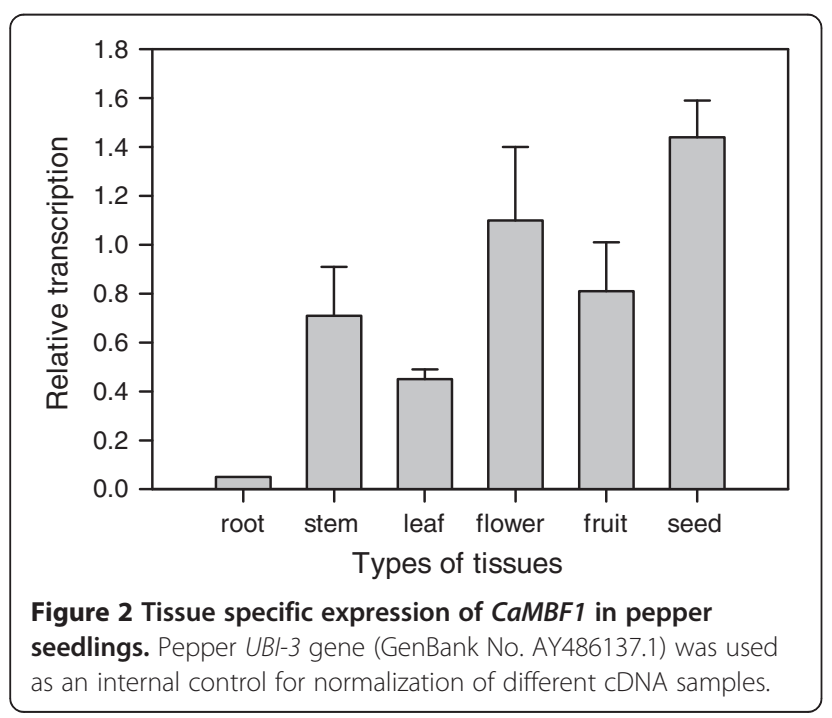

CaMBF1 gene has no obvious effect on AtMBF1s transcripts in Arabidopsis. The CaMBF1 gene was not detected in WT plants. CaMBF1 transcript in transgenic plants subjected to cold stress, salinity, and ABA was much lower than that detected in transgenic plants under normal conditions (Figure 6), suggesting that expression of CaMBF1 in Arabidopsis was dramatically decreased by stress treatments such as cold, salinity, and ABA. Furthermore, the visible symptoms of leaf damage in transgenic seedlings were observed to examine the tolerance of CaMBF1-expressing plants to cold stress. As shown in Figure 7, overexpression of the pepper CaMBF1 gene in Arabidopsis aggravated the visible symptoms of leaf damage caused by cold stress in seedlings. Wilting appeared after $6 \mathrm{~h}$ of cold stress in transgenic plants and became serious at $24 \mathrm{~h}$, while control leaves only exhibited withering after $48 \mathrm{~h}$ of cold stress. Meanwhile, to evaluate the extent of cell damage caused by cold stress in CaMBF1-expressing seedlings, electrolyte leakage was measured. The transgenic plants presented 1.5 folds higher electrolyte leakage than WT, which suggests that the membrane is likely to be impaired in these seedlings subjected to cold stress (Figure 8). These results suggested that overexpression of CaMBF1 in Arabidopsis could downregulate the expression of genes involved in stress tolerance.

We selected a group of candidate genes and conducted qRT-PCR analysis to test this hypothesis (Figure 9). Earlier studies have found RD29A, RD22, RAB18, KIN1 and $E R D 15$ to be involved in the response to dehydration and cold/ABA [20-23]. Compared with normal conditions, cold stress induced RD29A, ERD15 and KIN1 genes expression in both transgenic and WT plants (Figure 9A, D and E). After cold treatment, the expression of RD29A, ERD15 (except at $48 \mathrm{~h}$ ) and KIN1 in the transgenic plants was lower than that in the WT plants. Meanwhile, RAB18 

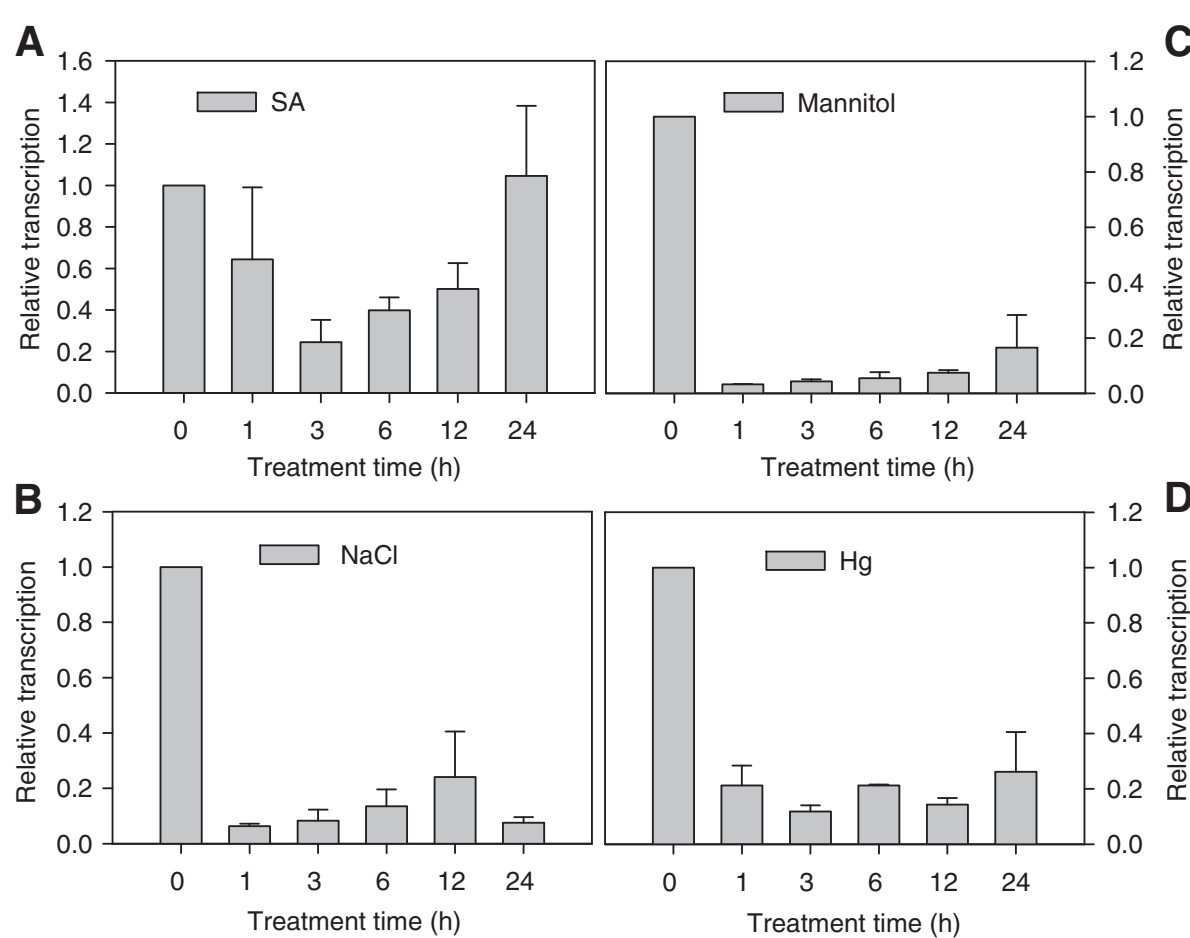

Figure 3 Analysis of CaMBF1 expression profiles in pepper seedlings in response to different stress treatments. The pepper seedlings were sprayed with $5 \mathrm{mM}$ SA solution (A); the pepper seedlings were exposed to salt stress (300 mM NaCl) (B), osmotic stress (300 mM mannitol) (C) and heavy metal (300 $\mu \mathrm{M} \mathrm{Hg}$ ) (D) for the indicated times (0, 1, 3, 6, 12 and $24 \mathrm{~h}$ ). Pepper UBl-3 gene (GenBank No. AY486137.1) was used as an internal control for normalization of different cDNA samples. The expression level of CaMBF1 at $0 \mathrm{~h}$ was used as control (quantities of calibrator) and was assumed as 1. Error bars represent standard error of means based on three independent reactions.

and $R D 22$ transcripts were dramatically decreased in both transgenic and control plants subjected to cold stress (Figure 9B and C). The expression of the RD22 gene was basically not detected in transgenic plants under cold stress; the decrease in $R A B 18$ expression in transgenic plants was similar to that in WT plants during
$24 \mathrm{~h}$ of cold stress. Overall, after cold treatment overexpression of the CaMBF1 gene in Arabidopsis suppressed chilling-induced RD29A, ERD15 and KIN1 transcripts and aggravated chilling-decreased $R D 22$ expression. Therefore, $C A M B F 1$ appeared to act as a negative regulator of stressresponsive gene expression such as RD29A, ERD15 KIN1
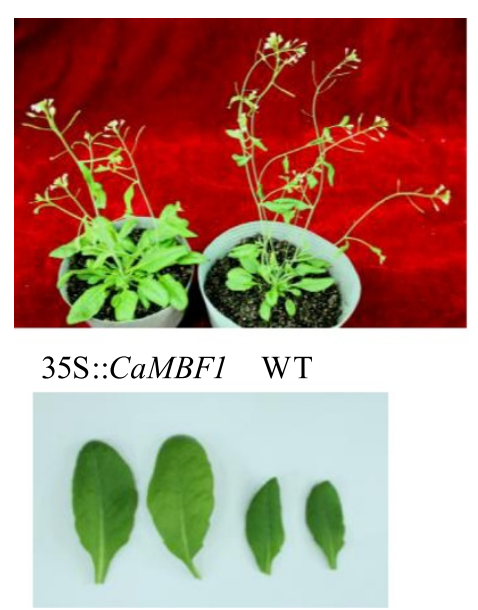

Figure 4 Phenotypic analysis of wild-type and CaMBF1-overexpressing transgenic Arabidopsis (\#12 and \# 21). Wild-type (Col-0) and transgenic Arabidopsis were grown at $22^{\circ} \mathrm{C}$, with a $14 / 10 \mathrm{~h}$ photoperiod, a light intensity of $120 \mathrm{mmol} \mathrm{m} \mathrm{s}^{-1}$, and $70 \%$ relative humidity. *indicates the least significant difference (LSD) test significant at $\mathrm{P}<0.05$.

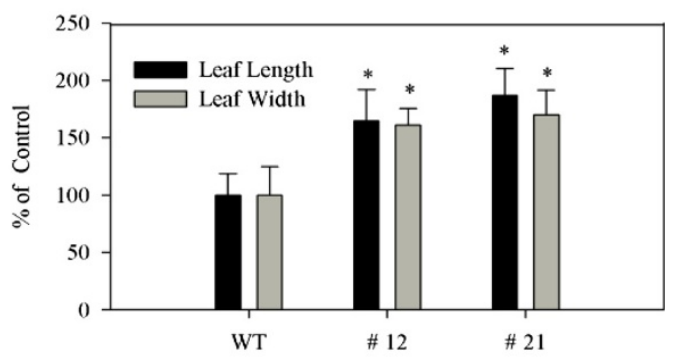




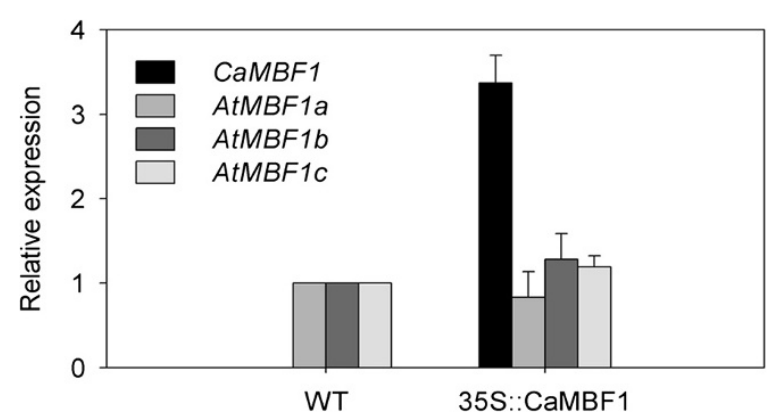

Figure 5 Relative expressions of AtMBF1s transcripts in transgenic or wild-type plants under normal growth conditions. Arabidopsis encodes three different AtMBF1 isoforms (AtMBF1a, At2g42680; AtMBF1b, At3g58680; AtMBF1C, At3g24500).

and $R D 22$, consistent with the results from leaf chilling injury assays and electrolyte leakage measurement.

\section{The CaMBF1-overexpressing Arabidopsis is hypersensitive} to salt stress

To further characterize the tolerance of CaMBF1-overexpressing plants to salinity, transgenic seeds were germinated in MS/2 media supplemented with $100 \mathrm{mM}$ $\mathrm{NaCl}$ and allowed to grow for 8 days. Transgenic seeds exhibited hypersensitivity to salinity compared with WT seeds (Figure 10A). On medium containing $100 \mathrm{mM}$ $\mathrm{NaCl}, 78 \%$ of WT seeds germinated within $2 \mathrm{~d}$, whereas the germination percentage for transgenic seeds was only $12 \%$ during the same period. In addition, the germination and subsequent growth of transgenic seedlings

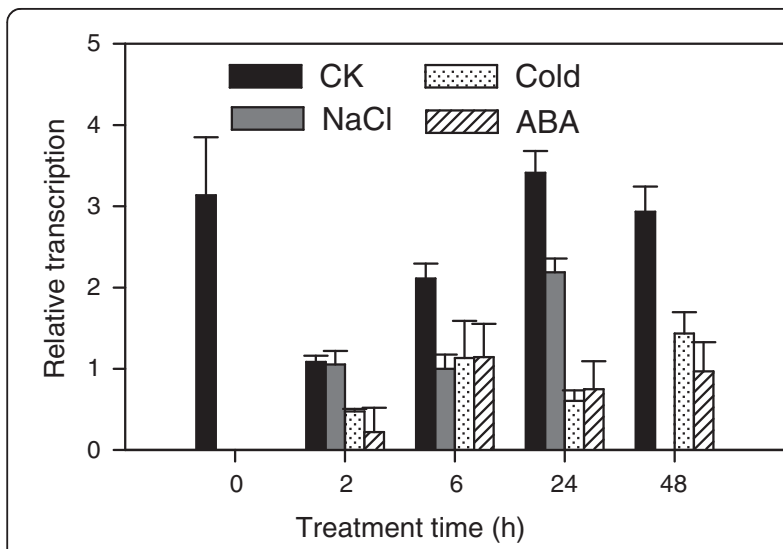

Figure 6 Analysis of CaMBF1 expression profiles in transgenic lines in response to different stress treatments. For salt stress and $A B A$ treatments, 2-week-old seedlings were submerged in a MS/2 medium containing $150 \mathrm{mM} \mathrm{NaCl}$ and $100 \mu \mathrm{M}$ ABA solutions, respectively. For cold treatment, 2-week-old transgenic seedlings were subjected to $4^{\circ} \mathrm{C}$ for $48 \mathrm{~h}$. Samples were collected from both stress-treated and control (CK) plants at 0, 2, 6, 24, and $48 \mathrm{~h}$ of cold, salt stress and ABA treatment. Arabidopsis elF4A gene (At3g13920) was used as an internal control for normalizing the variations in cDNA amounts used. Error bars represent standard error of means based on three independent reactions. were comparable to WT plants on normal medium, but were significantly more inhibited by salt stress (Figure 10). The cotyledons of 6-day-old transgenic lines were bleached 7 days after transfer to medium containing $150 \mathrm{mM} \mathrm{NaCl}$ and became serious at 9 days, whereas the cotyledons of WT plants were slightly affected (Figure 10B). On the other hand, the primary root growth of transgenic plants was similar to that of WT plants under salt stress. However, lateral root formation was more severely influenced by salinity in transgenic plants compared with WT plants (Figure 10B).

Similarly, comparative expression analyses of the stress gene markers described above were also performed by qRT-PCR on RNA isolated from 2-week-old plants grown under non-stress and salt stress conditions (Figure 11). Upon salinity treatment, several gene markers (RD29A, RAB18 and KIN1) were highly induced in both WT and transgenic seedlings (Figure 11A, B and E). Conversely, $R D 22$ and ERD15 transcripts were dramatically decreased in both transgenic and WT plants subjected to salt stress (Figure $11 \mathrm{C}$ and $\mathrm{D}$ ). Furthermore, the expression of RD29A, RAB18, KIN1 and ERD15 in the transgenic lines was higher than that in the WT plants under high salt conditions. Therefore, overexpression of CaMBF1 in Arabidopsis appeared to positively regulate the expression of stress-responsive gene markers such as RD29A, RAB18, KIN1 and ERD15, which was not consistent with the results from seed germination and cotyledon greening assays. In some cases, the level of stress gene expression appears to be insufficient to induce tolerance changes [24-26].

\section{Altered expression of stress-responsive HSPs in the CaMBF1-overexpressing Arabidopsis}

To evaluate whether $C a M B F 1$ expression could be correlated with alterations of other stress-responsive genes, classical heat-shock genes, HSP70 and HSP9O were tested in all lines by qRT-PCR (Figure 12). Compared with control plants, HSP70 and HSP9O transcripts (except at $0 \mathrm{~h}$ ) were decreased in transgenic plants under normal conditions. After cold treatment, the expression of HSP70 and HSP9O in the transgenic plants was lower than that in the WT plants (Figure 12A and B); whereas, the expression of these genes in the transgenic lines was higher than that in the WT plants under high salt conditions (Figure $12 \mathrm{C}$ and $\mathrm{D}$ ), indicating that comparative regulation of HSPS in response to CaMBF1 overexpression could be related to different stresses.

\section{Discussion}

Here, we report a putative transcription coactivator from pepper seedlings, the putative amino acid sequence of which was $95 \%$ and $80 \%$ identical to those of StMBF1 and AtMBF1b, respectively. Therefore, CaMBF1 could 

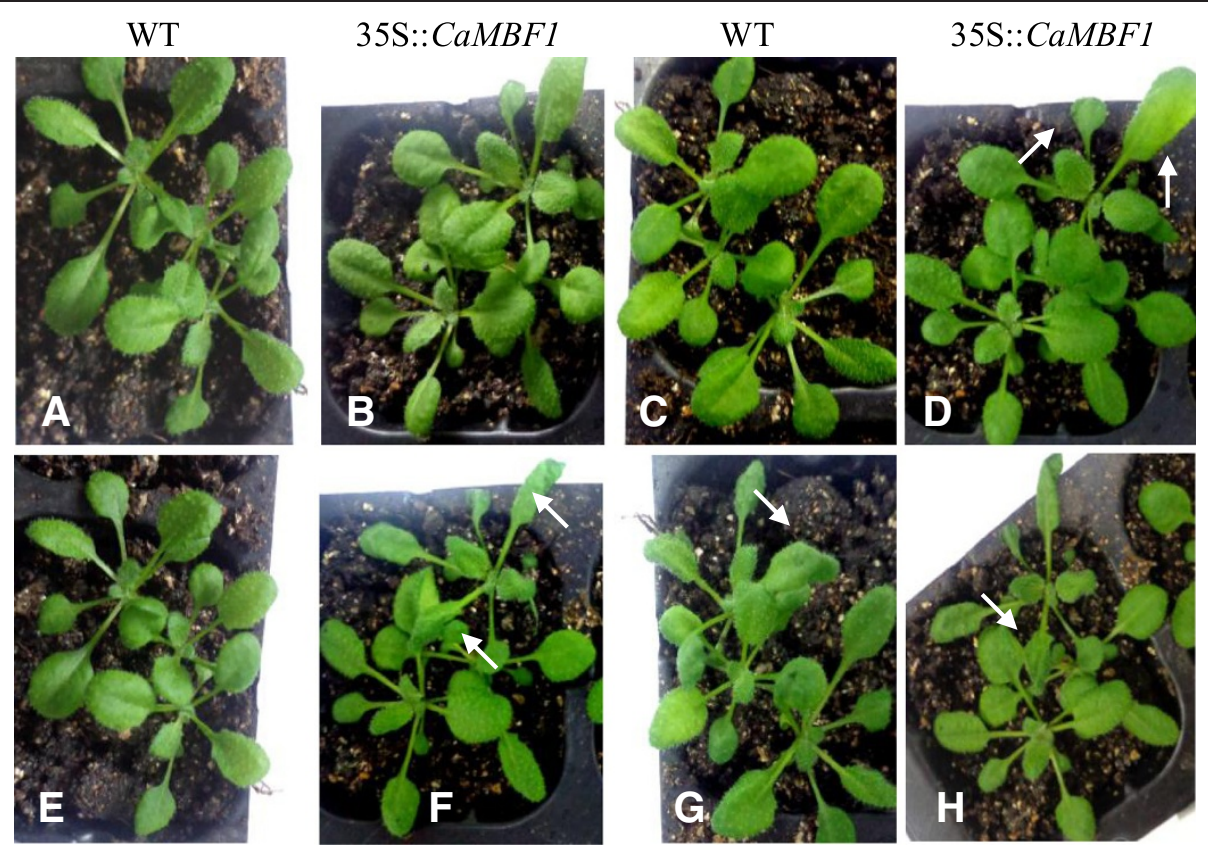

Figure 7 Effect of cold stress on visual damage symptoms of wild-type and CaMBF1-overexpressing transgenic plants. A, Wild-type Arabidopsis (Col-0) were subjected to cold stress for 2 h; B, Transgenic plants were subjected to cold stress for 2 h; C, Wild-type plants were subjected to cold stress for 6 h; D, Transgenic plants were subjected to cold stress for 6 h; $\mathbf{E}$, Wild-type plants were subjected to cold stress for 24 h; $\mathbf{F}$, Transgenic plants were subjected to cold stress for 24 h; G, Wild-type plants were subjected to cold stress for 48 h; $\mathbf{H}$, Transgenic plants were subjected to cold stress for $48 \mathrm{~h}$. The differences among treatments are marked with white arrows in rosette leaves. Photographs show plants subjected to cold stress for $48 \mathrm{~h}$.

be categorized as belonging to the same group as $S t M B F 1$ [8]. The deduced amino acid sequences of plant $M B F 1 s$ revealed the existence of highly conserved amino acid residues in each group [19]. Additionally, tissue-specific expression of CaMBF1 observed here (Figure 2) suggests that CaMBF1 may be involved in physiological processes of pepper plants. In this regard, the highly homologous StMBF1 also exhibits a ubiquitous tissue distribution [8].

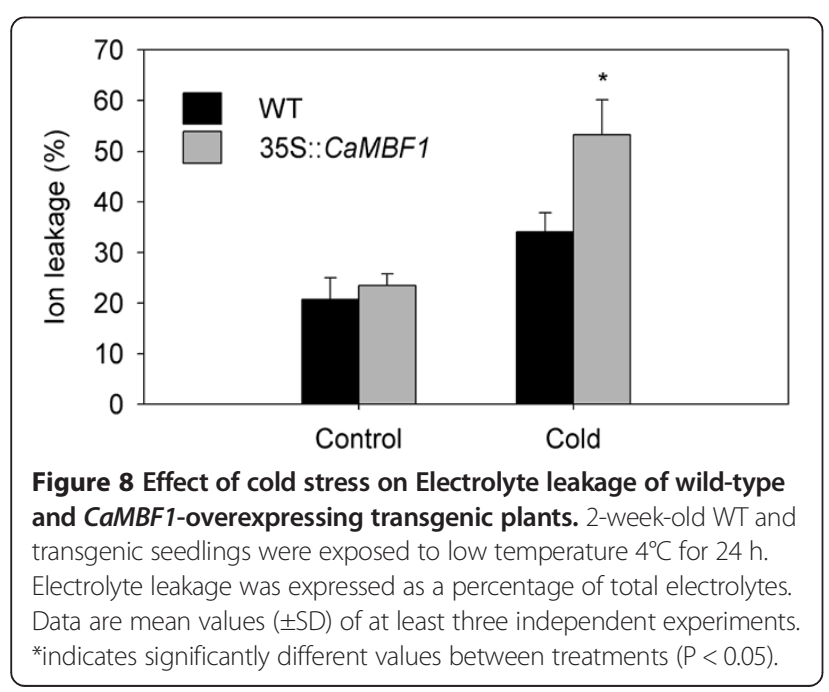

In the present study, CaMBF1 transcript in pepper or Arabidopsis seedlings was dramatically decreased in response to abiotic stresses such as $\mathrm{SA}, \mathrm{ABA}$, high salt, osmotic, and heavy metal stress (Figures 3 and 6). Particularly, under cold stress the expression of CaMBF1 was downregulated in Arabidopsis seedlings (Figure 6). These results indicated that $C a M B F 1$ may be negatively involved in stress signaling pathways. Unlike other $M B F 1$ genes, the expression of $A t M B F 1 c$ is induced by various stresses, including salinity, drought, heat, $\mathrm{H}_{2} \mathrm{O}_{2}$ and $\mathrm{ABA}$, and is not affected by cold stress [19]. Salinity also induced AtMBF1a/b expression [10] and cold stress did not significantly change mRNA accumulation of AtMBF1a and AtMBF1b in Arabidopsis [19].

CaMBF1-overexpressing plants showed extremely large leaf phenotypes (Figure 4). This finding could be explained by similar evidence reported by Tojo et al. [27] who suggested that AtMBF1s play a crucial role in controlling rapid leaf expansion through promotion of cell expansion. The amino acid sequences of $M B F 1 \mathrm{~s}$ are widely conserved among plant species. Similarly, transgenic Arabidopsis expressing AtMBF1c were 20\% larger than control plants and produced more seeds [9].

The visible symptoms of leaf damage in CaMBF1expressing transgenic Arabidopsis were observed more severely than that in WT plants (Figure 7) and the transgenic plants presented 1.5 folds higher electrolyte leakage than 

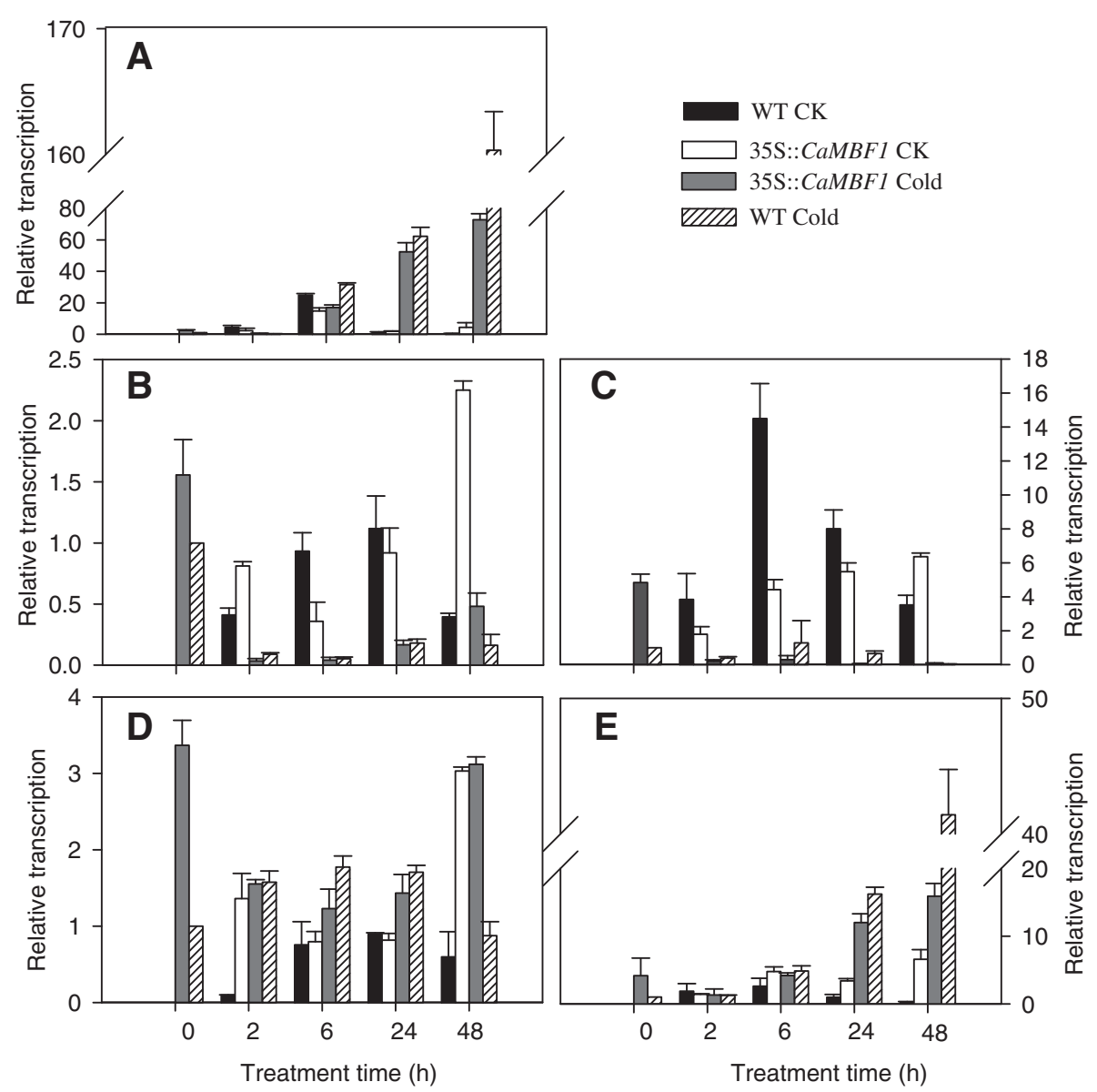

Figure 9 Expression of stress-responsive genes in wild-type and transgenic plants subjected to cold stress. Relative expression levels of stress-responsive genes were determined by qRT-PCR using CDNA synthesized from total RNAs isolated from the leaves of 2-week-old Arabidopsis exposed to low temperature $4^{\circ} \mathrm{C}$ for 48 h. A, RD29A; B, RAB18; C, RD22; D, ERD15; E, KIN1. There were four treatments: WT CK represents wild-type plants grown under non-stressed conditions; 35S::CaMBF1 CK represents transgenic plants grown under non-stressed conditions; 355::CaMBF1 Cold represents transgenic plants subjected to cold stress; WT Cold represents wild-type plants subjected to cold stress. Arabidopsis elF4A gene (At3g13920) was used as an internal control for normalization of different cDNA samples. The expression levels of stress-responsive genes in wild-type plants at $0 \mathrm{~h}$ were used as control (quantities of calibrator) and were assumed as 1. Three biological triplicates were averaged and Bars indicate standard error of the mean.

WT under cold stress (Figure 8), suggesting that the tolerance of transgenic plants to cold stress was reduced. This result was in agreement with the fact that some genes isolated from the reverse SSH library, including a MBF1 homologue, were related to reduction in cold tolerance of plants [17]. Moreover, overexpression of the CaMBF1 gene in Arabidopsis reduced the expression of RD29A, ERD15, KIN1, and RD22 during cold treatment (Figure 9). CaMBF1 may reduce the tolerance of Arabidopsis to cold stress by negatively regulating stress-tolerant gene expression. Suzuki et al. [9] reported that the tolerance of MBF1cexpressing transgenic seedlings to cold stress was similar to that of WT seedlings. On the other hand, CaMBF1expressing transgenic plants showed high susceptibility to salt stress imposed during seed germination (Figure 10A). In contrast to this result, the triple knock-down mutant $(a b c-)$ presented a significant diminution of germination under osmotic stress [28] and $M B F 1$ genes negatively regulated ABA-dependent inhibition of germination [29]. The cotyledons and lateral root formation were more severely influenced by salinity in transgenic plants compared with WT plants (Figure 10B). Meanwhile, root growth of MBF1a/c-expressing plants adopted to the high or lowsalt condition comparatively better than WT plants $[9,10]$. Seed germination is controlled by the antagonistic action of gibberellic acid (GA) or ethylene and ABA [30-32]. MBF1 may be involved in several hormone signal transduction pathways (ethylene, GA/ABA) during seed germination [6,33]. In addition, the expression of RD29A, RAB18, KIN1 and ERD15 in CaMBF1-expressing transgenic Arabidopsis was higher than that in WT plants under high salt conditions (Figure 11). Kim et al. [10] also reported that 


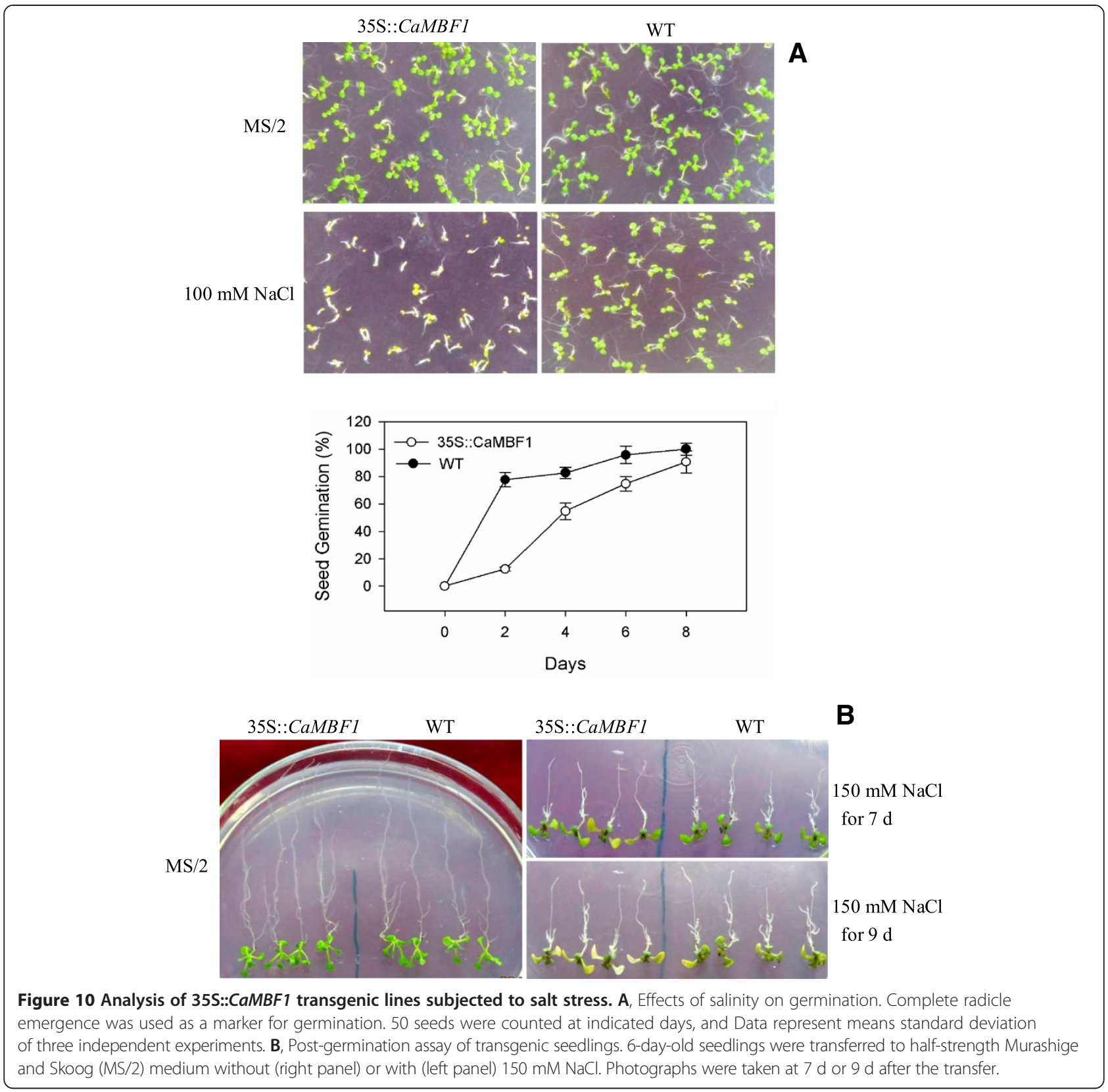

$M B F 1 a$-overexpressing transgenic Arabidopsis induced RD29A, ERD15, and KIN2 during the course of salt treatment. The accumulation of a number of defense transcripts was similarly augmented in $M B F 1 c$ transgenic Arabidopsis in response to heat stress [9].

The expression patterns of the above-mentioned stress gene markers in transgenic plants subjected to cold stress were different from those in transgenic lines under salt stress. This difference could be related to that each stress opens out specific defense mechanisms in young seedlings and the participation of CaMBF1 might be different depending on the stress condition imposed. Since different stresses may disrupt plant growth and development in specific ways, the plant might alleviate damage by different mechanisms. The results of this study, that overexpression of the pepper CaMBF1 gene differently modules the expression of HSPs in Arabidopsis under cold and salt stresses (Figure 12), supported this hypothesis. There were similar reports as follows: constitutive expression of stress-responsive HSP genes was augmented in the $a b c$ - mutant, indicating that AtMBF1s may act as negative regulators of HSP in Arabidopsis thaliana seedlings [28]. Suzuki et al. [9] described that transcripts encoding classical HSPs accumulated to a similar level in WT and transgenic plants over-expressing $M B F 1 c$; they suggested that the enhanced tolerance of 

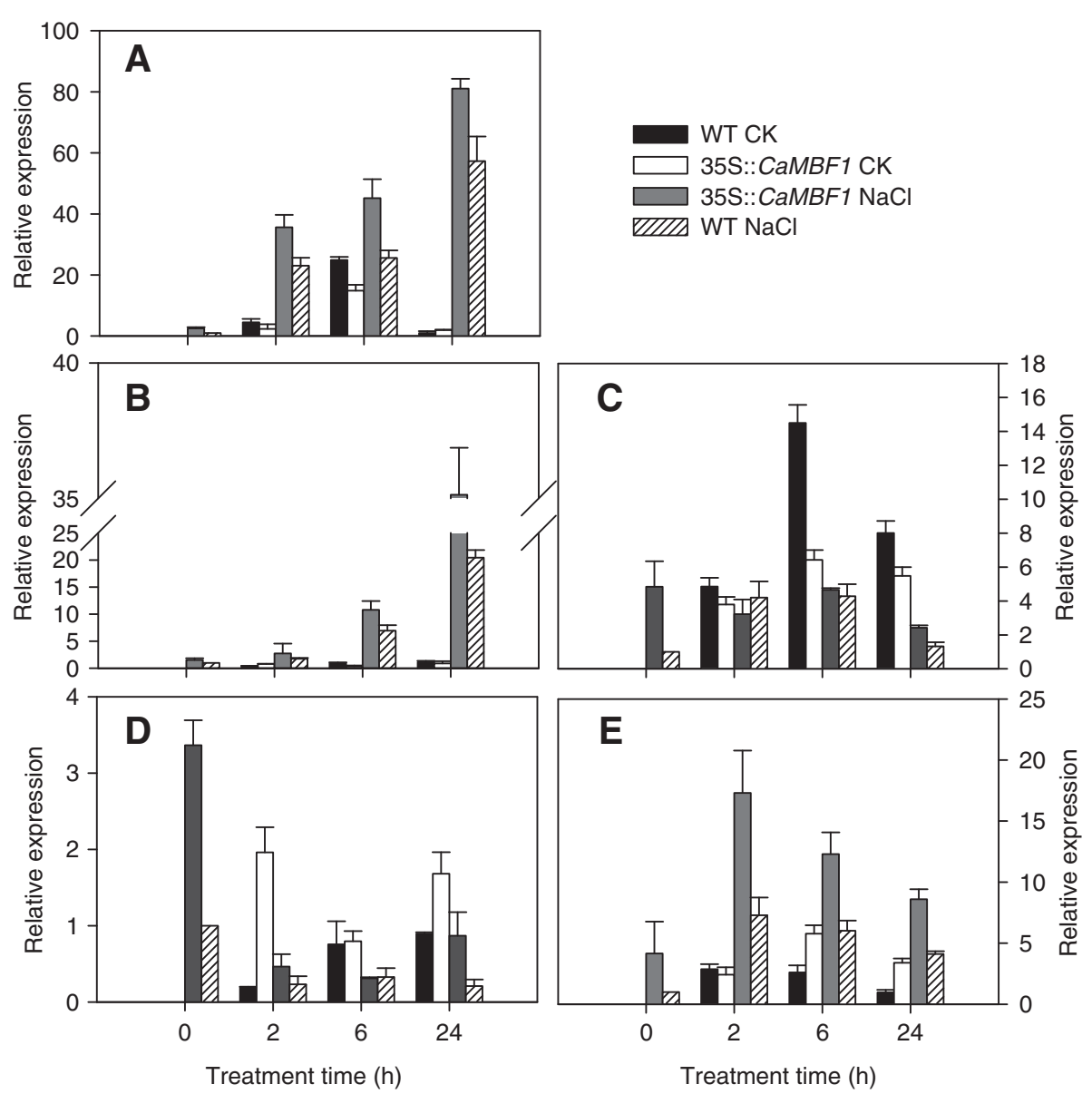

Figure 11 Expression of stress-responsive genes in wild-type and transgenic plants subjected to salt stress. Relative expression levels of stress-responsive genes were determined by qRT-PCR using CDNA synthesized from total RNAs isolated from the leaves of 2-week-old Arabidopsis subjected to high-salt stress (150 mM NaCl) for 24 h. A, RD29A; B, RAB18; C, RD22; D, ERD15; E, KIN1. There were four treatments: WT CK represents wild-type plants grown under non-stressed conditions; 35S::CaMBF1 CK represents transgenic plants grown under non-stressed conditions; 355 :.:CaMBF1 $\mathrm{NaCl}$ represents transgenic plants subjected to salt stress; WT NaCl represents wild-type plants subjected to salt stress. Arabidopsis elF4A gene (At3g13920) was used as an internal control for normalization of different cDNA samples. The expression levels of stress-responsive genes in wild-type plants at $0 \mathrm{~h}$ were used as control (quantities of calibrator) and were assumed as 1. Three biological triplicates were averaged and Bars indicate standard error of the mean.

these plants to osmotic and heat-shock stress was associated with the expression of other stress-responsive genes rather than with the constitutive expression of HSPs. Finally, our data together with previous evidences support that Capsicum annum CaMBF1 play a different role as Arabidopsis AtMBF1 in response to salt or cold stress. Further studies will be necessary to reveal specific functions for each gene.

\section{Conclusions}

This study demonstrates that the manipulation of the $C a M B F 1$ gene from pepper using a transgenic approach can lead to reduced cold-stress and salt-stress tolerance in Arabidopsis. In addition, overexpression of CaMBF1 may reduce stress tolerance by downregulating stressresponsive genes to aggravate the leaf damage caused by cold stress. However, upregulation of such stressresponsive genes appears to be insufficient to induce tolerance of $C a M B F 1$ transgenic plants to salt stress. The CaMBF1 gene could be a candidate gene for future research on abiotic stress signaling pathways and genetic engineering of novel pepper cultivars. The results of this study will be helpful in providing beneficial information to support biotechnology applications and molecular breeding, which clarify the function of a gene involved in abiotic stress in plants.

\section{Methods}

\section{Plant materials and stress treatments}

Pepper (Capsicum annuum L.) cv. P70 seeds were sown at a depth of $1.0 \mathrm{~cm}$ into 9-cm-deep plastic pots filled with growth medium consisting of grass charcoal and 

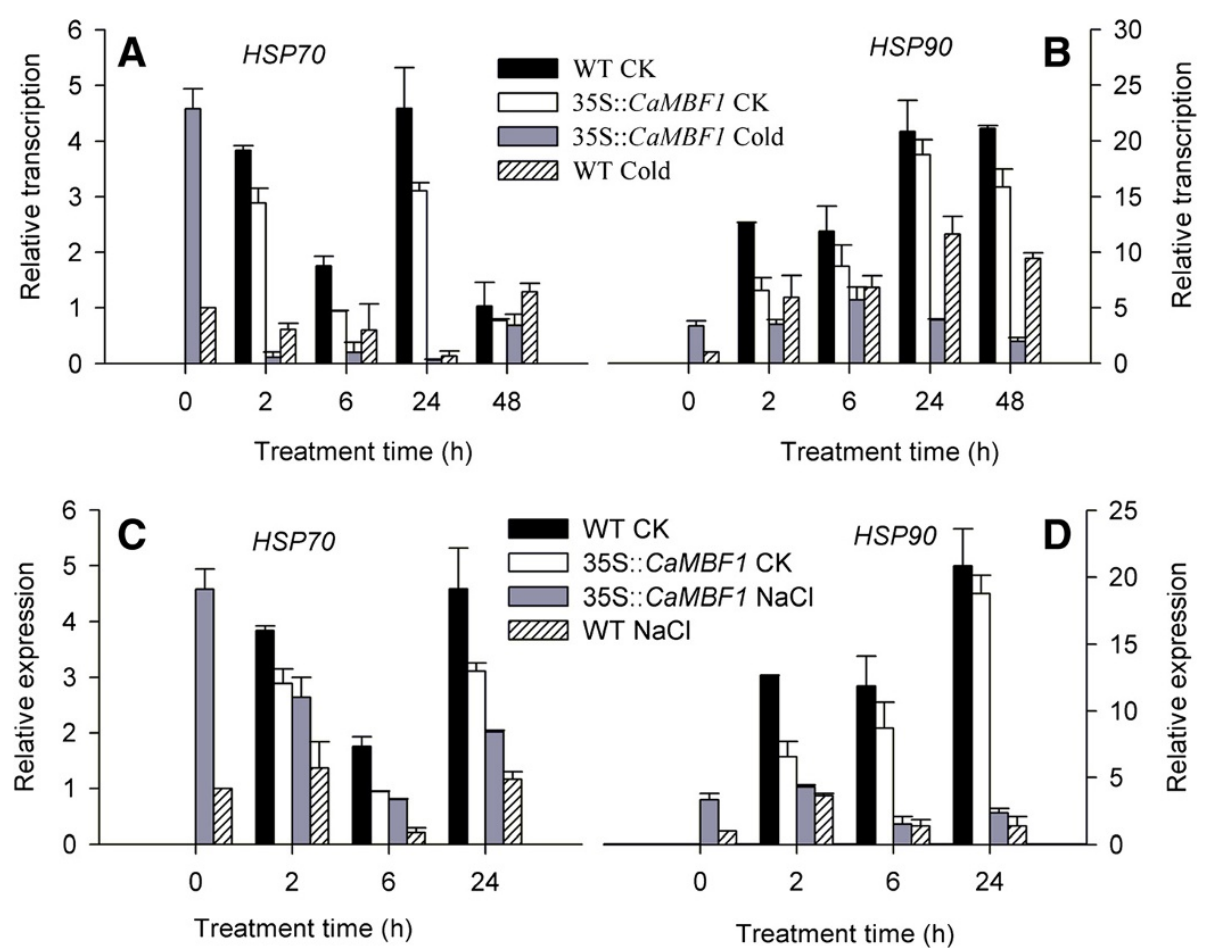

Figure 12 Expression of HSPs in wild-type and transgenic plants subjected to cold and salt stresses. Relative expression levels of stress-responsive genes were determined by qRT-PCR using CDNA synthesized from total RNAs isolated from the leaves of 2-week-old Arabidopsis subjected to cold stress for $48 \mathrm{~h}$ and high-salt stress for $24 \mathrm{~h}$ as described above, respectively. A, HSP70 from Arabidopsis under cold stress; $\mathbf{B}$, HSP9O from Arabidopsis under cold stress; C, HSP70 from Arabidopsis under salt stress; D, HSP90 from Arabidopsis under salt stress.

perlite in a ratio of 3:1 after accelerated germination and grown in a growth chamber using a previously described method [16]. The seedlings at the sixth leaf expansion stage were used to establish the following treatments. ABA and cold treatments were performed as described by Guo et al. [17]. For ABA and cold treatments, seedlings were sprayed with freshly prepared $0.57 \mathrm{mM}$ ABA solution or water (control). At $72 \mathrm{~h}$ after foliar application, control and ABA treatment groups were subjected to chilling stress at $6^{\circ} \mathrm{C}$. For salt, osmotic, and the heavy metal $(\mathrm{Hg})$ treatments, the seedling roots were immersed in solutions containing $300 \mathrm{mM}$ sodium chloride $(\mathrm{NaCl}), 300 \mathrm{mM}$ mannitol, or $300 \mu \mathrm{M} \mathrm{Hg}$ and maintained at $25^{\circ} \mathrm{C}$ for the indicated times. For SA treatment, seedlings were sprayed with $5 \mathrm{mM}$ SA solution and incubated for the indicated times. The treated seedlings were harvested after $0,1,3,6,12$ and $24 \mathrm{~h}$ for examination of CaMBF1 expression pattern under various stress conditions. At each time point, two or three upper young leaves from four separate seedlings were collected to form one sample, wrapped with foil, immediately frozen in liquid nitrogen and stored at $-80^{\circ} \mathrm{C}$. The treatments were arranged in a randomized complete block design with three replicates.
Isolation of CaMBF1 CDNA clone and sequence analysis The MBF1-homologous EST (GenBank No: JZ198811) characterized from the differential screening of a coldrelated pepper seedling cDNA library was reported by Guo et al. [17]. The full-length open reading frame of the $M B F 1$ homologue was obtained using the cDNA fragment of this homolog as a probe by a homology-based candidate gene method [34]. The full-length forward and reverse primers for CaMBF1 were 5'-GAAGAAAAAAA GCAATGAGTGG-3' and 5'-GCAGAAACGAATTTA G-GATTTG-3' respectively. The theoretical molecular weight $(\mathrm{Mw})$ and isoelectric point $(\mathrm{pI})$ were calculated with the ExPASy compute $\mathrm{pI} / \mathrm{Mw}$ tool [35]. Sequence data were analyzed using Clustal W [36]. Homology searches in database were carried out using the default parameters of the BLAST program on the website http://www.ncbi.nlm.nih.gov:blast [37].

\section{Generation of CaMBF1 transgenic Arabidopsis plants}

Full-length forward and reverse primers with an added $B a m H I$ site were used to generate a DNA fragment encoding the CaMBF1 gene. The CaMBF1 fragment was inserted into the cloning site of the pMD19 T-vector (Takara, Tokyo, Japan) and then this plasmid DNA was digested using XbaI and BamHI from the pMD19 
T-vector. The CaMBF1 DNA fragment was inserted into the XbaI-BamHI site of the pVBG2307 vector under the control of the $35 \mathrm{~S}$ cauliflower mosaic virus (CaMV) promoter, resulting in the pVBG2307-CaMBF1 construct. The pVBG2307 vector was constructed according to pCAMBIA2300 vector [38]. This construct was confirmed by sequencing and then introduced into Agrobacterium tumefaciens GV3101 using electroporation. Arabidopsis (ecotype Columbia-0, Col-0), chosen for transgenic studies, was grown in a controlled environment chamber at $22^{\circ} \mathrm{C}$, with a $14 / 10 \mathrm{~h}$ photoperiod, a light intensity of $120 \mathrm{mmol} \mathrm{m}^{-2} \mathrm{~s}^{-1}$, and $70 \%$ relative humidity. Transgenic plants were generated by Agrobacterium-mediated transformation using the floral dip method [39]. CaMBF1-overexpressing transgenic seedlings were confirmed by examining the segregation ratio of the kanamycin selectable marker and by PCR analysis of NPTII and CaMBF1 using the primers NPTII-F/R and CaMBF1-F/R (Additional file 1: Table S1). T2 lines that produced $100 \%$ kanamycin -resistant plants in the T3 generation were considered as homozygous transformants. In each experiment, $\mathrm{T} 2$ generations of homozygous transgenic lines (\#5, \#12 and \#21) were selected for further analysis. Similar phenotypes and results used for this study were observed in more than three independent lines of transgenic plants.

\section{Performance of transgenic lines under stress treatments}

Two-week-old transgenic seedlings were subjected to various treatments. Cold treatment was conducted in the dark by exposure of plants grown on vermiculite soil at $22^{\circ} \mathrm{C}$ to $4^{\circ} \mathrm{C}$ for $48 \mathrm{~h}$, whereas control plants were placed in the dark at $22^{\circ} \mathrm{C}$ for $48 \mathrm{~h}$. After cold treatment, wild-type (WT) and CaMBF1-overexpressing transgenic plants were visually examined to determine the extent of chilling damage. For high-salinity and ABA treatments, 2-weekold seedlings were submerged in half-strength Murashige and Skoog (MS/2) medium containing $150 \mathrm{mM}$ $\mathrm{NaCl}$ or $100 \mu \mathrm{M}$ ABA solutions, whereas control plants were submerged in a MS/ 2 medium. Third-fourth rosette leaves were collected from both stress-treated and control plants after $0,2,6,24$, and $48 \mathrm{~h}$ of cold, salt stress or ABA treatments. At each time point, sample was frozen in liquid nitrogen, stored at $-80^{\circ} \mathrm{C}$ and used for extraction of total RNA. The treatments were arranged in a randomized complete block design with three replicates.

Homozygous T2 seeds of the transgenic lines were used for phenotypic analysis. For high-salinity treatment, seeds of WT and transgenic plants were plated on MS/2 agar plates supplemented with $100 \mathrm{mM} \mathrm{NaCl}$, grown in a growth chamber, and assessed for percentage of germination after various times (0, 2, 4, 6 and $8 \mathrm{~d})$. Experiments were done in triplicate for each line (50 seeds each). 6 -day-old plants grown on normal MS/2 agar plates were transferred to vertical MS/2 agar plates containing $150 \mathrm{mM} \mathrm{NaCl}$, and grown for another week as previously described [40]. The root growth and cotyledon greening of 24 seedlings were observed.

\section{Measurement of electrolyte leakage}

Leaflets from 2-week-old seedlings were transferred to $4^{\circ} \mathrm{C}$ and incubated for $24 \mathrm{~h}$ in the dark in the growth chamber. The conductivity of the suspending solution was measured according to the method of Arce et al. [28]. The electrical conductivity of the solution was measured using an electrical conductivity analyzer (DDS-307; Shanghai Precision Scientific Instrument Co., Ltd., China) before and after autoclaving at $120^{\circ} \mathrm{C}$ for $30 \mathrm{~min}$ to release the total electrolytes. The conductivity was scored at least for 4 plants per line and pretreatment. Electrolyte leakage was expressed as a percentage of total electrolytes.

\section{Real-time quantitative PCR (qRT-PCR) analysis}

RNA extraction, cDNA preparation and qRT-PCR were performed as described by Guo et al. [17]. Relative gene expression levels were determined using the $2^{\wedge}-\Delta \Delta$ CT method. Total RNA was extracted from the leaves of pepper plants subjected to various stress for $0,1,3,6,12$, and $24 \mathrm{~h}$ as described above. The ubiquitin -conjugating protein gene (UBI-3, GenBank accession no. AY486137.1) from pepper plants was amplified as a reference gene for normalization of CaMBF1 cDNA samples. On the other hand, total RNA of CaMBF1 transgenic and WT Arabidopsis were used to examine the expression of seven stress-related genes (RD29A, RAB18, ERD15, KIN1, RD22, HSP70 and HSP90) and three Arabidopsis isoforms (AtMBF1a, AtMBF1b, AtMBF1c). Arabidopsis eIF4A gene (At3g13920) was included in the assays as an internal control for normalizing the variations in cDNA amounts used [41]. The corresponding specific primers were listed in Additional file 1: Table S1.

\section{Statistical analysis}

Data were analyzed using analysis of variance (SAS 8.2, North Carolina State University, USA) and mean separation was analyzed using the least significant difference. The $\mathrm{P}$ value $<0.05$ was considered to be significant.

\section{Supporting data}

All the supporting data are included as additional files.

\section{Additional file}

\section{Additional file 1: Table S1. The sequences of primers used in this} study.

\section{Competing interests}

The authors declare that they have no competing interests. 


\section{Authors' contributions}

WLG, RGC and ZHG conceived and designed the experiments; WLG, XHD, $Z Z, Y X Y$ and GYW performed the experiments; $Z Z$ and $Y X Y$ analyzed the data; ZHG contributed reagents/materials/analysis tools; WLG wrote the paper. All authors read and approved the final manuscript.

\section{Acknowledgements}

The authors thank Xin-Ling Zhang for assistance with Arabidopsis transformation. This work was supported by the National Natural Science Foundation of China (\#31201615, \#31272163), "The Twelfth Five-Year" Plan of National Science and Technology in Rural Areas (\#2011BAD12B03) and the Shaanxi Provincial Science and Technology Coordinating Innovative Engineering Project (\#2012KTCL02-09) and the Henan Provincial commodity vegetable industry technology system (\#S2010-03-G06).

\section{Author details}

'College of Horticulture, Northwest A\&F University, Yangling, Shaanxi, P R China. ${ }^{2}$ State Key Laboratory of Crop Stress Biology in Arid Areas, Northwest A\&F University, Yangling, Shaanxi, P R China. ${ }^{3}$ School of Horticulture Landscape Architecture, Henan Institute of Science and Technology, Xinxiang, Henan, P R China.

Received: 17 March 2014 Accepted: 12 May 2014

Published: 20 May 2014

\section{References}

1. Li FQ, Ueda H, Hirose S: Mediators of activation of fushi tarazu gene transcription by BmFTZ-F1. Mol Cell Biol 1994, 14:3013-3021.

2. Roeder RG: The complexities of eukaryotic transcription initiation: regulation of preinitiation complex assembly. Trends Biochem Sci 1991, 16:402-408.

3. Takemaru K, Harashima S, Ueda H, Hirose S: Yeast co-activator MBF1 mediates GCN4-dependent transcriptional activation. Mol Cell Biol 1998, 18:4971-4976

4. Jindra M, Gaziova I, Uhlirova M, Okabe M, Hiromi Y, Hirose S: Coactivator MBF1 preserves the redox-dependent AP-1 activity during oxidative stress in Drosophila. EMBO J 2004, 23:3538-3547.

5. Tsuda K, Tsuji T, Hirose S, Yamazaki K: Three Arabidopsis MBF1 homologs with distinct expression profiles play roles as transcriptional co-activators. Plant Cell Physiol 2004, 45:225-231.

6. Hommel M, Khali-Ahmad Q, Jaimes-Miranda F, Mila I, Pouzet C, Latché A, Pech JC, Bouzayen M, Regad F: Over-expression of a chimeric gene of the transcriptional co-activator MBF1 fused to the EAR repressor motif causes developmental alteration in Arabidopsis and tomato. Plant Sci 2008, 175:168-177.

7. Arce DP, Tonón C, Zanetti ME, Godoy AV, Hirose S, Casalongué CA: The potato transcriptional co-activator StMBF1 is up-regulated in response to oxidative stress and interacts with the TATA-box binding protein. J Biochem Mol Biol 2006, 39(4):355-60.

8. Godoy AV, Zanetti ME, San Segundo B, Casalongue CA: Identification of a putative Solanum tuberosum transcriptional coactivator up-regulated in potato tubers by Fusarium solani f. sp. eumartii infection and wounding Physiol Plant 2001, 112:217-222

9. Suzuki N, Rizhsky L, Liang H, Shuman J, Shulaev V, Mittler R: Enhanced tolerance to environmental stress in transgenic plants expressing the transcriptional co-activator multiprotein bridging factor 1c. Plant Physiol 2005, 139:1313-1322.

10. Kim MJ, Lim GH, Kim ES, Ko CB, Yang KY, Jeong JA, Lee MC, Kim CS: Abiotic and biotic stress tolerance in Arabidopsis overexpressing the multiprotein bridging factor 1a (MBF1a) transcriptional coactivator gene. Biochem Biophys Res Commun 2007, 354(2):440-446.

11. Mateos RM, Bonilla-Valverde D, del Rio LA, Palma JM, Corpas FJ: NADP-dehydrogenases from pepper fruits: effect of maturation. Physiol Plantarum 2008, 135(2):130-139.

12. Nguyen HT, Leipner J, Stamp P, Guerra-Peraza O: Low temperature stress in maize (Zea mays L.) induces genes involved in photosynthesis and signal transduction as studied by suppression subtractive hybridization. Plant Physiol Biochem 2009, 47:116-122.

13. Korkmaz A, Korkmaz Y, Demirkıran AR: Enhancing chilling stress tolerance of pepper seedlings by exogenous application of 5-aminolevulinic acid. Environ Exp Bot 2010, 67:495-501.
14. Airaki M, Leterrier M, Mateos RM, Valderrama R, Chaki M, Barroso JB, LA D r, Palma JM, Corpas FJ: Metabolism of reactive oxygen species and reactive nitrogen species in pepper (Capsicum annuum L.) plants under low temperature stress. Plant Cell Environ 2012, 35:281-295.

15. Pressman E, Shaked R, Firon N: Exposing pepper plants to high day temperatures prevents the adverse low night temperature symptoms. Physiol Plantarum 2006, 126:618-626.

16. Guo WL, Chen RG, Gong ZH, Yin YX, Ahmedand SS, He YM: Exogenous abscisic acid increases antioxidant enzymes and related gene expression in pepper (Capsicum annuum) leaves subjected to chilling stress. Genet Mol Res 2012, 11(4):4063-4080.

17. Guo WL, Chen RG, Gong ZH, Yin YX, Li DW: Suppression subtractive hybridization analysis of genes regulated by application of exogenous abscisic acid in pepper plant (Capsicum annuum L.) leaves under chilling stress. PloS One 2013, 8(6):e66667. doi:10.1371/journal. pone.0066667.

18. Rizhsky I, Liang H, Mittler R: The combined effect of drought stress and heat shock on gene expression in tobacco. Plant Physiol 2002, 130:1143-1151.

19. Tsuda K, Yamazaki K: Structure and expression analysis of three subtypes of Arabidopsis MBF1 genes. Biochim Biophys Acta 2004, 1680:1-10.

20. Yamaguchi-Shinozaki K, Shinozaki K: The plant hormone abscisic acid mediates the drought-induced expression but not the seed-specific expression of rd22, a gene responsive to dehydration stress in Arabidopsis thaliana. Mol Gen Genet 1993, 238:17-25.

21. Yamaguchi-Shinozaki K, Shinozaki K: A novel cis-acting element in an Arabidopsis gene is involved in responsiveness to drought, lowtemperature, or high-salt stress. Plant Cell 1994, 6:251-264.

22. Kiyosue T, Yamaguchi-Shinozaki K, Shinozaki K: ERD15, a cDNA for a dehydration-induced gene from Arabidopsis thaliana. Plant Physiol 1994, 106:1707.

23. Cheong YH, Sung SJ, Kim BG, Pandey GK, Cho JS, Kim KN, Luan S: Constitutive overexpression of the calcium sensor CBL5 confers osmotic or drought stress tolerance in Arabidopsis. Mol Cells 2010, 29:159-165.

24. Kim KN, Cheong YH, Grant JJ, Pandey GK, Luan S: CIPK3, a calcium sensor-associated protein kinase that regulates abscisic acid and cold signal transduction in Arabidopsis. Plant Cell 2003, 15:411-423.

25. Pandey GK, Cheong YH, Kim KN, Grant JJ, Li L, Hung W, D'Angelo C, Weinl S, Kudla J, Luan S: The calcium sensor calcineurin B-like 9 modulates abscisic acid sensitivity and biosynthesis in Arabidopsis. Plant Cell 2004, 16:1912-1924.

26. Pandey GK, Grant JJ, Cheong YH, Kim BG, Li L, Luan S: ABR1, an APETALA2domain transcription factor that functions as a repressor of $A B A$ response in Arabidopsis. Plant Physiol 2005, 139:1185-1193.

27. Tojo T, Tsuda K, Yoshizumi T, Ikeda A, Yamaguchi J, Matsui M, Yamazaki K: Arabidopsis MBF1s control leaf cell cycle and its expansion. Plant Cell Physiol 2009, 50:254-64.

28. Arce DP, Godoy AV, Tsuda K, Yamazaki K, Valle EM, Iglesias MJ, Mauro MFD, Casalongué CA: The analysis of an Arabidopsis triple knock-down mutant reveals functions for MBF1genes under oxidative stress conditions. J Plant Physiol 2010, 167:194-200.

29. Mauro MFD, Iglesias MJ, Arce DP, Valle EM, Arnold RB, Tsuda K, Yamazaki K Casalongué CA, Godoy AV: MBF1s regulate ABA-dependent germination of Arabidopsis seeds. Plant Signal Behav 2012, 7(2):188-192.

30. Bewley JD: Seed germination and dormancy. Plant Cell 1997, 9:1055-1066.

31. Beaudoin N, Serizet C, Gosti F, Giraudat J: Interactions between abscisic acid and ethylene signaling cascades. Plant Cell 2000, 12(7):1103-1115.

32. Cheng $\mathrm{WH}$, Chiang MH, Hwang SG, Lin PC: Antagonism between abscisic acid and ethylene in Arabidopsis acts in parallel with the reciprocal regulation of their metabolism and signaling pathways. Plant Mol Biol 2009, 71:61-80.

33. Kucera B, Cohn MA, Leubner-Metzger G: Plant hormone interactions during seed dormancy release and germination. Seed Sci Res 2005, 15:281-307.

34. Zhu W, Lu MH, Gong ZH, Chen RG: Cloning and expression of a small heat shock protein gene CaHSP24 from pepper under abiotic stress. Afr J Biotechnol 2011, 10(25):4968-4976.

35. Bjellqvist B, Hughes GJ, Pasquali C, Paquet N, Ravier F, Sanchez JC, Frutiger S, Hochstrasser D: The focusing positions of polypeptides in immobilized $\mathrm{pH}$ gradients can be predicted from their amino acid sequences. Electrophoresis 1993, 14:1023-1031.

36. Thompson JD, Higgins DG, Gibson TJ: CLUSTAL W: Improving the sensitivity of progressive multiple sequence alignment through 
sequence weighting, position-specific gap penalties and weight matrix choice. Nucleic Acids Res 1994, 22:4673-4680.

37. Altschul SF, Madden TL, Schaffer AA, Zhang JH, Zhang Z, Miller W, Lipman DJ: Gapped BLAST and PSI-BLAST: a new generation of protein database search programs. Nucleic Acids Res 1997, 25:3389-3402.

38. Ahmed SS, Gong ZH, Ji JJ, Yin YX, Xiao HJ, Khan MA, Rehman A, Ahmad l: Construction of the intermediate vector pVBG2307 by incorporating vital elements of expression vectors pBI121 and pBI221. Genet Mol Res 2012, 11(3):3091-3104

39. Clough SJ, Andrew FB: Floral dip: a simplified method for Agrobacteriummediated transformation of Arabidopsis thaliana. Plant J 1998, 16(6):735-743.

40. Lee S, Seo PJ, Lee HJ, Park CM: A NAC transcription factor NTL4 promotes reactive oxygen species production during drought-induced leaf senescence in Arabidopsis. Plant I 2012, 70:831-844.

41. Gutierrez L, Mauriat M, Gue'nin S, Pelloux J, Lefebvre JF, Louvet R, Rusterucc C, Moritz T, Guerineau F, Bellini C, Van Wuytswinkel O: The lack of a systematic validation of reference genes: a serious pitfall undervalued in reverse transcription-polymerase chain reaction (RT-PCR) analysis in plants. Plant Biotechnol J 2008, 6:609-618.

doi:10.1186/1471-2229-14-138

Cite this article as: Guo et al:: Reduced tolerance to abiotic stress in transgenic Arabidopsis overexpressing a Capsicum annuum multiprotein bridging factor 1. BMC Plant Biology 2014 14:138.

\section{Submit your next manuscript to BioMed Central and take full advantage of:}

- Convenient online submission

- Thorough peer review

- No space constraints or color figure charges

- Immediate publication on acceptance

- Inclusion in PubMed, CAS, Scopus and Google Scholar

- Research which is freely available for redistribution 